\title{
CD98 expression is associated with the grade of malignancy in thymic epithelial tumors
}

\author{
KYOICHI KAIRA ${ }^{1}$, TOSHIAKI TAKAHASHI ${ }^{1}$, MASATO ABE ${ }^{2}$, HIROAKI AKAMATSU ${ }^{1}$, \\ KAZUO NAKAGAWA ${ }^{3}$, YASUHISA OHDE ${ }^{3}$, TAKEHIRO OKUMURA ${ }^{3}$, HARUYASU MURAKAMI ${ }^{1}$, \\ ASUKA TSUYA ${ }^{1}$, YUKIKO NAKAMURA ${ }^{1}$, TATEAKI NAITO ${ }^{1}$, HARUHIKO KONDO $^{3}$, \\ TAKASHI NAKAJIMA ${ }^{2}$, MASAHIRO ENDO ${ }^{4}$ and NOBUYUKI YAMAMOTO ${ }^{1}$ \\ Divisions of ${ }^{1}$ Thoracic Oncology, ${ }^{2}$ Diagnostic Pathology, ${ }^{3}$ Thoracic Surgery, and ${ }^{4}$ Diagnostic Radiology, \\ Shizuoka Cancer Center, 1007 Shimonagakubo Nagaizumi-cho, Sunto-gun, Shizuoka 411-8777, Japan
}

Received May 5, 2010; Accepted June 29, 2010

DOI: 10.3892/or_00000931

\begin{abstract}
CD98 has been associated with tumor growth and is highly expressed in human neoplasms. The aim of this study was to evaluate the clinicopathological significance of CD98 expression in thymic epithelial tumors. Forty-nine patients with thymic epithelial tumors were included in this study. Tumor sections were stained by immunohistochemistry for CD98; vascular endothelial growth factor (VEGF); microvessels (CD31 and CD34); cell cycle control marker (p53); and apoptosis marker (Bcl-2). CD98 expression for low-risk thymomas, high-risk thymomas, and thymic carcinomas were $1(4 \%)$ of $27,9(82 \%)$ of 11 , and $11(100 \%)$ of 11 , respectively. There was positive correlation between CD98 and VEGF $(\mathrm{p}<0.001)$, microvessel density $(\mathrm{CD} 31$ and CD34) $(\mathrm{p}<0.001)$ and p53 $(\mathrm{p}<0.001)$. However, Bcl-2 showed no positive correlation with CD98 expression. The expression of CD98 were also significantly associated with the grade of malignancy in thymic epithelial tumors. Multivariate analysis revealed that overexpression of CD98 was a significant independent factor predicting a poor outcome in thymic epithelial tumors. CD98 expression was associated with the grade of malignancy in thymic epithelial tumors. Moreover, CD98 expression was closely correlated with angiogenesis and cell cycle control, and was useful for predicting poor outcome.
\end{abstract}

\section{Introduction}

Thymic epithelial tumors are broadly classified into thymoma and thymic carcinoma. Thymic tumors represent $0.2-1.5 \%$ of

Correspondence to: Dr Kyoichi Kaira, Division of Thoracic Oncology, Shizuoka Cancer Center, 1007 Shimonagakubo Nagaizumi-cho, Sunto-gun, Shizuoka 411-8777, Japan E-mail: kkaira1970@yahoo.co.jp

Key words: CD98, thymic epithelial tumor, angiogenesis, cell cycle, apoptosis all malignancies, overall it is an uncommon malignancy with an incidence of 0.15 cases per 100,000 populations (1). Tumors of the thymus are a heterogeneous group of tumors, ranging from relatively benign thymomas to highly aggressive carcinomas. The World Health Organization (WHO) published a new histological classification of thymic epithelial tumors into 3 subgroups, low-risk thymomas (types A, AB, and B1), high-risk thymomas (types B2 and B3), and thymic carcinomas $(2,3)$.

CD98 is a disulphide-linked $125-\mathrm{kDa}$ heterodimeric membrane glycoprotein, which is found on the cell surface of most normal cells. It was first identified on the cell surface of T lymphocytes (4). However, CD98 has been shown to be involved in the cellular proliferation, transformation, fusion, and adhesion and also in the L-type amino acid transport (LAT) system, in addition to regulating integrin activation, and therefore integrin signaling and anchorage-independent growth. CD98 is reconstituted and expressed at high levels on the surface of many types of tumor cells. The overexpression of CD98 has been shown to result in cellular transformation $(5,6)$. Recent studies demonstrated that CD98 expression is associated with tumor progression and metastasis in human neoplasms (7-12). CD98 expression is increased in a variety of carcinomas, including adenocarcinoma, transitional cell carcinoma, squamous cell carcinoma and endometrial carcinoma $(13,14)$. However, the expression of CD98 in thymic epithelial tumors has not previously been investigated.

To elucidate the role of CD98 expression in thymic epithelial tumors, we undertook an immunohistochemical examination of CD98 expression in primary sites. In addition, CD98 was correlated with angiogenic markers such as expression of vascular endothelial growth factor (VEGF) and microvessel density (MVD), cell cycle control (p53) and apoptosis (Bcl-2).

\section{Materials and methods}

Patients. Between October 2002 and March 2009, 52 consecutive patients with thymic epithelial tumors were analyzed in the study. Of these patients, 3 patients were excluded for 
further studies because the tissue specimens were not available. Thus, a total of 49 patients ( 23 men, 26 women) were eligible in the study. The study protocol was approved by the institutional review board.

The patient characteristics are listed in Table I. The age of the patients ranged from 32 to 80 years (median age, 64 years). Thirty-eight of the 49 patients underwent surgical resection and the remaining 11 underwent only percutaneous needle-core biopsy. All tumors were staged according to the Masaoka classification (15). In patients with surgical excision, the stage was determined by operative and pathological findings. In patients with biopsy only, the stage was determined by diagnostic imaging including computed tomography and $2-\left[1^{8} \mathrm{~F}\right]$-fluoro-2-deoxy-D-glucose positron emission tomography $\left({ }^{18} \mathrm{~F}-\mathrm{FDG} \mathrm{PET}\right)$. All surgical specimens were reviewed and classified according to the WHO classification $(2,3)$ by an experienced lung pathologist who was unaware of clinical or imaging findings. For patients without surgery, the histological classification was determined by specimens obtained through needle-core biopsy. As the initial treatment, 38 patients were treated with surgery, 8 chemotherapy and 3 thoracic irradiation. The tumor size of primary tumors ranged from 15 to $140 \mathrm{~mm}$ (median, $60 \mathrm{~mm}$ ). All tumors were regrouped into 3 subgroups: low-risk thymomas (types A, AB, and B1), high-risk thymomas (types $\mathrm{B} 2$ and B3), and thymic carcinomas.

Immunohistochemical staining. Immunohistochemical staining was performed according to the procedure described in previous reports $(12,16-19)$. The following antibodies were used: a goat polyclonal antibody against CD98 (Santa Cruz Biotechnology, Inc., 1:200 dilution); a monoclonal antibody against VEGF (Immuno-Biological Laboratories Co., Ltd., Japan, 1:100 dilution); a mouse monoclonal antibody against CD31 (Dako, 1:50 dilution); a mouse monoclonal antibody against CD34 (Nichirei, Tokyo, Japan, 1:800 dilution); a mouse monoclonal antibody against p53 (D07; Dako, 1:50 dilution); a mouse monoclonal antibody against Bcl-2 (Dako, 1:100 dilution).

The expression of CD98 was considered positive if distinct membrane staining was present. For CD98, a semiquantitative scoring method was used: $1,<10 \% ; 2,10-25 \%$; $3,25-50 \% ; 4,51-75 \%$; and $5,>75 \%$ of cells positive. The tumors in which stained tumor cells made up $>25 \%$ of the sample were graded as positive.

The expression of VEGF was quantitatively assessed according to the percentage of immunoreactive cells in a total of 1,000 neoplastic cells. The number of CD31- and CD34positive vessels was counted in four selected hot spots in a $x 400$ field $\left(0.26 \mathrm{~mm}^{2}\right.$ field area). MVD was defined as the mean count of microvessels per $0.26 \mathrm{~mm}^{2}$ field area $(18,19)$.

For p53, microscopic examination for the nuclear reaction product was performed and scored. According to previous reports $(16,20,21), \mathrm{p} 53$ expression in $>10 \%$ of tumor cells was defined as high expression. Expression of Bcl-2 was considered to be positive when there was staining of area of the epithelial component of the tumor. Sections were assessed using a light microscope in a blinded fashion by at least two of the authors.
Table I. Patient characteristics in thymic epithelial tumors.

\begin{tabular}{lc}
\hline Parameter & No. of patients \\
\hline Total & 49 \\
Age (years) & $64(32-80)$ \\
$\quad$ Median, range & \\
Sex & $23 / 26$ \\
$\quad$ Male/Female & $60(15-140)$ \\
Tumor size (mm) & \\
$\quad$ Median, range & \\
WHO classification & $5 / 17 / 5 / 6 / 5 / 11$ \\
$\quad$ A/AB/B1/B2/B3/thymic carcinoma & \\
Masaoka classification & $14 / 15 / 8 / 5 / 7$ \\
I/II/III/Iva/IV & \\
Initial treatment after diagnosis & 38 \\
Surgery & 3 \\
Radiation & 8 \\
Chemotherapy & \\
\hline
\end{tabular}

Statistical analysis. Probability values of $<0.05$ indicated a statistically significant difference. Fisher's exact test was used to examine the association of two categorical variables. Correlation of different variables was analyzed using the non-parametric Spearman's rank test. The Kaplan-Meier method was used to estimate survival as a function of time, and survival difference were analyzed by the log-rank test. Multivariate analyses were performed using stepwise Cox proportional hazards model to identify independent prognostic factors. Statistical analysis was performed using JMP 8 for Windows (SAS, Institute Inc., Cary, NC, USA).

\section{Results}

Immunohistochemical analysis. CD98, VEGF, CD31, CD34, p53 and Bcl-2 immunohistochemical staining was evaluated for the 49 primary lesions (Fig. 1).

CD98 immunostaining was detected in carcinoma cells in tumor tissues and localized predominantly on their plasma membrane. The cytoplasmic staining was rarely evident. In the present study, no expression of CD98 protein was observed in any normal epithelial cells of the lung, including bronchial epithelial and alveolar cells. A positive CD98 expression was recognized in $43 \%(21 / 49)$. The average score of the CD98 expression was $2.5 \pm 0.5$ on a scale of 1-5. CD98 expression for low-risk thymomas, high-risk thymomas, and thymic carcinomas were $1(4 \%)$ of 27,9 $(82 \%)$ of 11 , and $11(100 \%)$ of 11 , respectively. Table II shows the scoring of CD98 expression according to WHO classification. The staining pattern of VEGF was uniformly localized in the cytoplasm and/or membrane of neoplasia. The median rate of VEGF positivity was $18 \%$ (range, 2-80\%), and the value of 18 was chosen as a cut-off point. High 
A

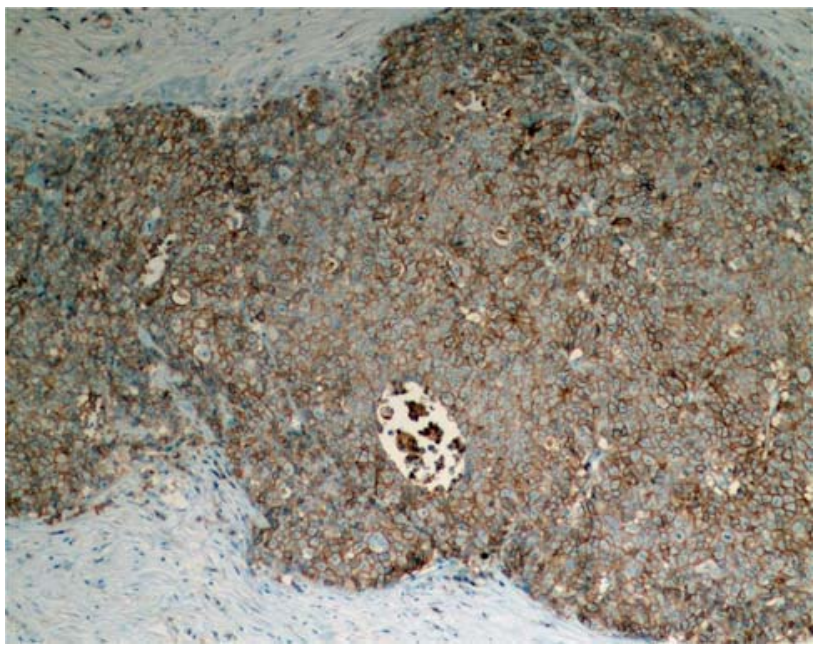

C

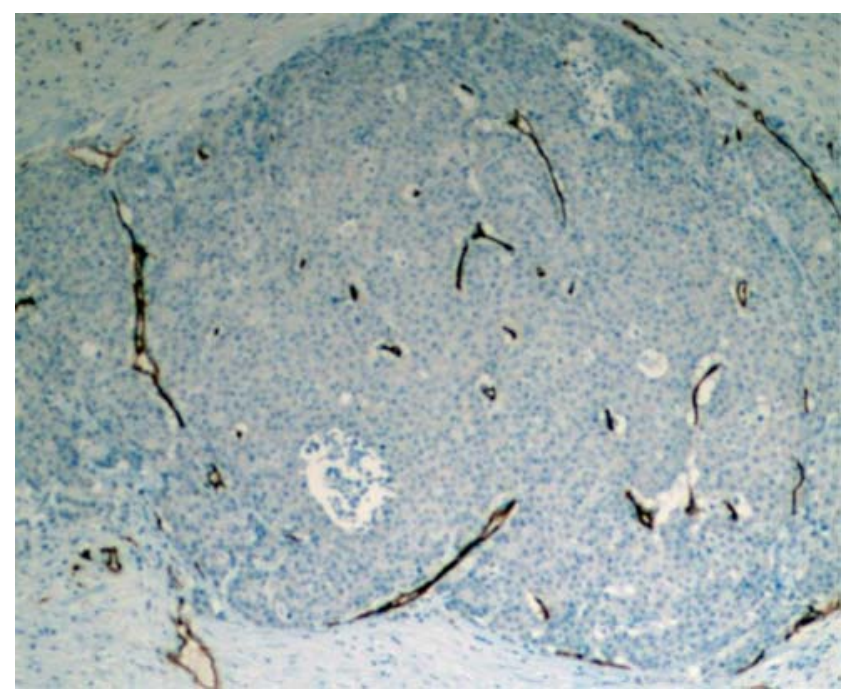

B

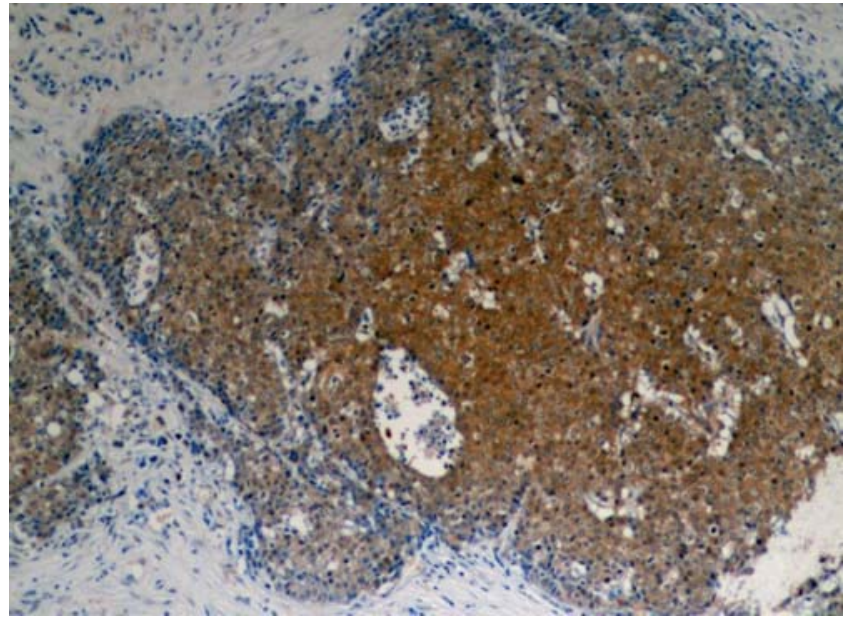

D

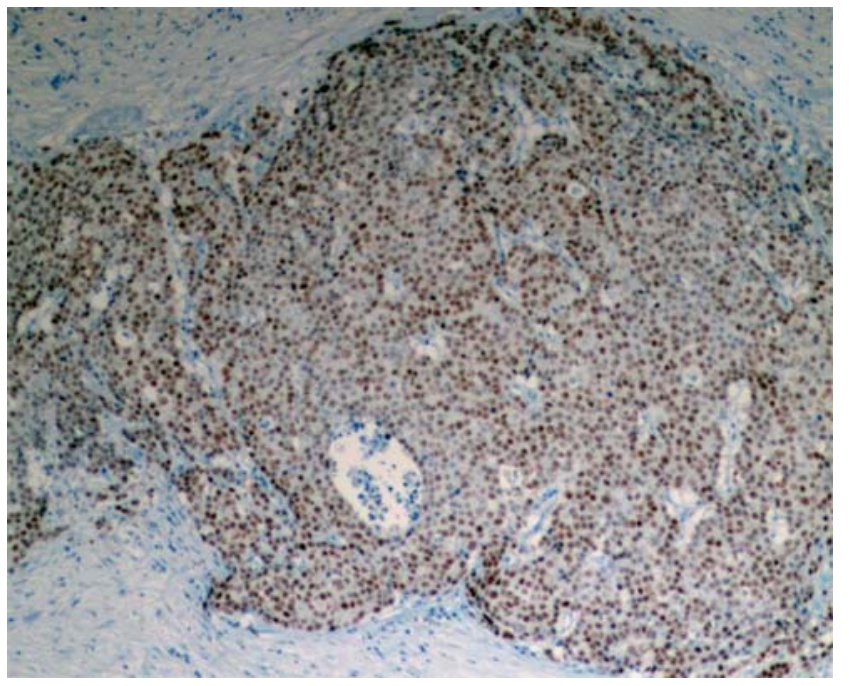

Figure 1. Immunohistochemical analysis of a resected primary tumor in a 46-year old man with thymic carcinoma: comparison of CD98, VEGF, CD34 and p53. The immunohistochemical score of CD98 was grade 5, and its immunostaining pattern was membranous (A). Immunostaining for vascular endothelial growth factor (VEGF). More than 75\% of tumor cells showed a positive reaction for anti-VEGF antibody (B). Immunostaining for CD34. Many small vessels positive for CD34 were seen in the stroma of the tumor tissue (C). Immunostaining pattern for p53 was nuclear staining (D).

Table II. Immunohistochemistry for CD98 expression according to WHO classification.

\begin{tabular}{llllll}
\hline $\begin{array}{l}\text { WHO } \\
\text { classification } \\
\text { (no. of patients) }\end{array}$ & 1 & 2 & 3 & 4 & 5 \\
\cline { 2 - 6 } & 1 & 4 & 0 & 0 & 0 \\
A (n=5) & 7 & 9 & 1 & 0 & 0 \\
AB $(n=17)$ & 2 & 3 & 0 & 0 & 0 \\
B1 $(n=5)$ & 0 & 2 & 4 & 0 & 0 \\
B2 $(n=6)$ & 0 & 0 & 4 & 1 & 0 \\
B3 $(n=5)$ & 0 & 0 & 4 & 3 & 4 \\
Thymic carcinoma $(n=11)$ & & & & &
\end{tabular}

expression was recognized in $43 \%$. The median numbers of CD31 and CD34 were $21(1-46)$ and 28 (8-45), respectively, and the values of cut-off point were 21 and 28 , respectively. High expression of CD31 and CD34 was seen in 39 and $43 \%$, respectively. VEGF expression was statistically associated with the number of microvessel count [CD31-MVD $(\gamma=0.6660, p<0.0001)$ and CD34-MVD $(\gamma=0.6993$, $\mathrm{p}<0.0001)$ ], respectively. High expression of p53 and Bcl-2 was recognized in 31 and $55 \%$, respectively.

Next, we examined a quantitative analysis of CD98 expression according to WHO classification (Fig. 2). The mean scoring of CD98 showed significant differences between low-risk thymomas and high-risk thymomas, and between high-risk thymomas and thymic carcinomas.

Relationship between CD98 expression and different variables. The results of the statistical comparison between CD98 expression and different variables in thymic epithelial tumors are listed in Table III. Gender, Masaoka staging, histology of thymic carcinoma, VEGF, MVD and p53 yield a statistically significant positive correlation. The results in 


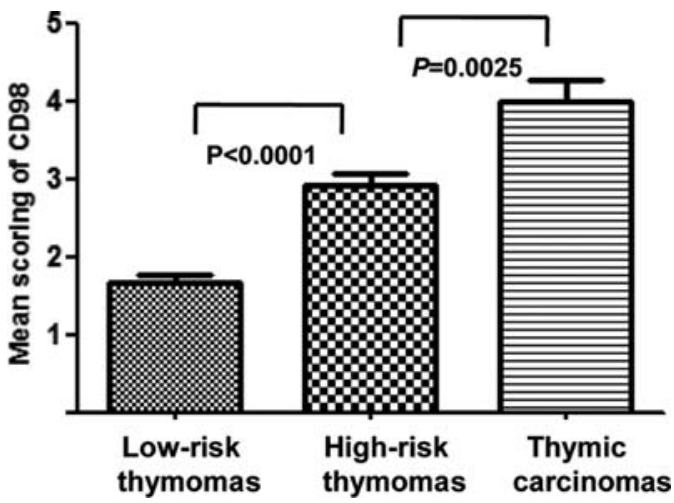

Figure 2. CD98 expression in a simplified WHO classification. Mean scoring of $\mathrm{CD} 98$ was $1.6 \pm 0.1$ in low-risk thymomas, $2.9 \pm 0.2$ in high-risk thymomas and $4.0 \pm 0.3$ in thymic carcinomas, demonstrating significant differences between the three groups (low-risk vs. high-risk, $\mathrm{p}<0.0001$; high-risk vs. thymic carcinoma, $\mathrm{p}=0.0025$ ).

A

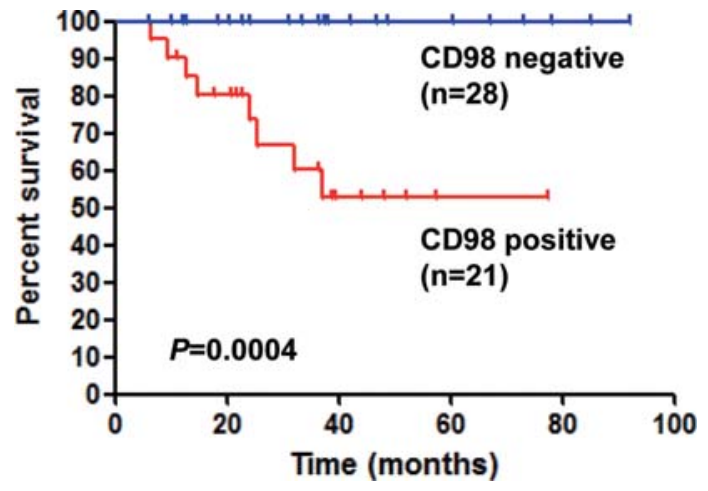

B

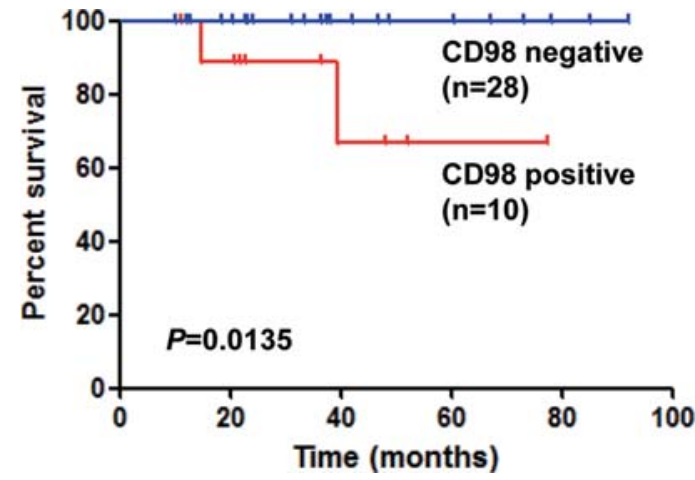

Figure 3. Survival in thymic epithelial tumors $(n=49)$ and thymomas $(n=38)$ according to CD98 expression. In thymic epithelial tumors, the 5-year survival rates of the CD98 positive and CD98 negative patients were 52.3 and $100 \%$, respectively, $(\mathrm{p}=0.0004)(\mathrm{A})$. In thymoma, the 5 -year survival rates of the CD98 positive and CD98 negative patients were 66.7 and $100 \%$, respectively, $(\mathrm{p}=0.0135)(\mathrm{B})$.

thymomas are listed in Table IV. Masaoka staging, VEGF, MVD and p53 yield a statistically significant positive correlation, and tumor size was significantly larger in CD98 negative than $\mathrm{CD} 98$ positive.

Survival analysis. Median follow-up time for all patients was 38.5 months (range, 6.2-85.1 months). The 5-year survival rate was $82.4 \%$, and the median survival time was
Table III. Relationship between CD98 expression and different variables in thymic epithelial tumors.

\begin{tabular}{|c|c|c|c|}
\hline \multirow[b]{2}{*}{ Parameter } & \multicolumn{3}{|c|}{ CD98 expression } \\
\hline & $\begin{array}{c}\text { Negative } \\
(n=28)\end{array}$ & $\begin{array}{l}\text { Positive } \\
(\mathrm{n}=21)\end{array}$ & P-value \\
\hline \multicolumn{4}{|l|}{ Age (years) } \\
\hline$\leq 65$ & 20 & 14 & 0.762 \\
\hline$>65$ & 8 & 7 & \\
\hline \multicolumn{4}{|l|}{ Gender } \\
\hline Male & 8 & 16 & 0.001 \\
\hline Female & 20 & 5 & \\
\hline \multicolumn{4}{|l|}{ Tumor size (mm) } \\
\hline$\leq 60$ & 7 & 11 & 0.073 \\
\hline$>60$ & 21 & 10 & \\
\hline \multicolumn{4}{|l|}{ Histology } \\
\hline Thymoma & 28 & 10 & $<0.001$ \\
\hline Thymic carcinoma & 0 & 11 & \\
\hline \multicolumn{4}{|l|}{$\begin{array}{l}\text { Masaoka } \\
\text { classification }\end{array}$} \\
\hline $\mathrm{I} / \mathrm{II}$ & 25 & 3 & $<0.001$ \\
\hline III/IV & 5 & 16 & \\
\hline \multicolumn{4}{|l|}{ VEGF } \\
\hline High & 3 & 18 & $<0.001$ \\
\hline Low & 25 & 3 & \\
\hline \multicolumn{4}{|l|}{ CD31 } \\
\hline High & 4 & 15 & $<0.001$ \\
\hline Low & 24 & 6 & \\
\hline \multicolumn{4}{|l|}{ CD34 } \\
\hline High & 4 & 17 & $<0.001$ \\
\hline Low & 24 & 4 & \\
\hline \multicolumn{4}{|l|}{ p53 } \\
\hline High & 2 & 13 & $<0.001$ \\
\hline Low & 26 & 8 & \\
\hline \multicolumn{4}{|l|}{ Bcl-2 } \\
\hline High & 16 & 11 & 0.778 \\
\hline Low & 12 & 10 & \\
\hline
\end{tabular}

VEGF, vascular endothelial growth factor.

not reached. In thymic epithelial tumors $(\mathrm{n}=49)$, the 5 -year survival rates of the CD98 positive and $\mathrm{CD} 98$ negative patients were 52.3 and $100 \%$, respectively, $(\mathrm{p}=0.0004)$ (Fig. 3A). In thymoma $(\mathrm{n}=38)$, the 5 -year survival rates of the CD98 positive and CD98 negative patients were 66.7 and $100 \%$, respectively, ( $\mathrm{p}=0.0135$ ) (Fig. 3B). Univariate and multivariate analysis of factors which affected the overall survival was performed (Table V). Multivariate analysis revealed that thymic carcinoma and CD98 expression were 
Table IV. Relationship between CD98 expression and different variables in thymoma.

\begin{tabular}{|c|c|c|c|}
\hline \multirow[b]{2}{*}{ Parameter } & \multicolumn{3}{|c|}{ CD98 expression } \\
\hline & $\begin{array}{l}\text { Negative } \\
(n=28)\end{array}$ & $\begin{array}{l}\text { Positive } \\
(n=10)\end{array}$ & P-value \\
\hline \multicolumn{4}{|c|}{ Age (years) } \\
\hline$\leq 65$ & 20 & 7 & 1.000 \\
\hline$>65$ & 8 & 3 & \\
\hline \multicolumn{4}{|l|}{ Gender } \\
\hline Male & 8 & 6 & 0.126 \\
\hline Female & 20 & 4 & \\
\hline \multicolumn{4}{|c|}{ Tumor size (mm) } \\
\hline$\leq 60$ & 7 & 8 & 0.006 \\
\hline$>60$ & 21 & 2 & \\
\hline \multicolumn{4}{|c|}{$\begin{array}{l}\text { Masaoka } \\
\text { classification }\end{array}$} \\
\hline I/II & 25 & 3 & 0.0018 \\
\hline III/IV & 5 & 5 & \\
\hline \multicolumn{4}{|l|}{ VEGF } \\
\hline High & 3 & 7 & $<0.001$ \\
\hline Low & 25 & 3 & \\
\hline \multicolumn{4}{|l|}{ CD31 } \\
\hline High & 4 & 5 & 0.004 \\
\hline Low & 24 & 5 & \\
\hline \multicolumn{4}{|l|}{ CD34 } \\
\hline High & 4 & 6 & 0.009 \\
\hline Low & 24 & 4 & \\
\hline \multicolumn{4}{|l|}{ p53 } \\
\hline High & 2 & 5 & 0.008 \\
\hline Low & 26 & 5 & \\
\hline \multicolumn{4}{|l|}{$\mathrm{Bcl}-2$} \\
\hline High & 16 & 4 & 0.468 \\
\hline Low & 12 & 6 & \\
\hline
\end{tabular}

VEGF, vascular endothelial growth factor.

significant risk factors for overall survival. The hazard ration of positive-expression of CD98 compared with negativeexpression was 2.730 (98\% confidence interval 1.122-12.01).

\section{Discussion}

The present study evaluated the clinical significance of CD98 expression in thymic epithelial tumors. The results of the study demonstrated that expression of CD98 tended to increase from low-grade to high grade thymic epithelial tumors. CD98 expression was closely correlated with angiogenesis and cell cycle control, and was useful for predicting poor outcome. Moreover, the overexpression of CD98 was
Table V. Univariate and multivariate survival analysis.

\begin{tabular}{lcc}
\hline & \multicolumn{2}{c}{ P-value } \\
\cline { 2 - 3 } Parameter & Univariate & Multivariate \\
\hline $\begin{array}{l}\text { Age } \\
(\leq 65 />65)\end{array}$ & 0.3439 & 0.8136 \\
$\begin{array}{l}\text { Gender } \\
(\text { Male/Female })\end{array}$ & 0.0634 & 0.5192 \\
$\begin{array}{l}\text { Histology } \\
\text { (Thymoma/Thymic carcinoma) }\end{array}$ & $<0.001$ & 0.0240 \\
$\begin{array}{l}\text { CD98 expression } \\
\text { (Positive/Negative) }\end{array}$ & $<0.001$ & 0.0094 \\
\hline
\end{tabular}

a significant independent factor to predict a poor outcome in thymic epithelial tumors.

In the present study, we found that overexpression of CD98 has an important role to play in the progression and development of thymic epithelial tumors. It is possible that CD98 has some influence on tumor cell growth. Recent studies demonstrated that positive CD98 expression was a significantly independent factor to predict poor prognosis in non-small cell lung cancer (NSCLC) (8-10). Moreover, we described that CD98 expression was closely correlated with L-type amino acid transporter 1 (LAT1) expression, and a cooperative expression of CD98 and LAT1 was significantly related with poor outcome in NSCLC $(10,11)$. LAT1 is widely expressed in primary human cancers and several cancer cell lines, where it has been shown to play essential roles in growth and survival (22). LAT1 requires covalent association with CD98 for its functional expression in plasma membrane (23). In thymic epithelial tumors, LAT1 is frequently expressed in thymic carcinomas but is absent in thymomas, and LAT1 expression was statistically associated with VEGF and MVD in thymic carcinomas (19). LAT1 expression has an important role to play in the development of thymic carcinomas. However, it is unknown whether LAT1 expression is associated with the progression and development of thymomas. In the present study, CD98 is expressed in thymomas, demonstrating significant difference between thymomas and thymic carcinomas. Moreover, CD98 expression is significantly higher in high-risk thymomas than low-risk thymomas, and positive CD98 expression is closely related with poor outcome in patients with thymomas. Therefore, CD98 expression may have an important role on the tumor cell growth in not only thymic carcinomas but also thymomas. To our knowledge, however, this is the first time that overexpression of CD98 is reported in thymic epithelial tumors. This study does not include data which we have presented previously (19). Further research to confirm these results and to explore the role of CD98 in thymic epithelial tumors is, therefore warranted.

Angiogenesis is essential for tumor growth and an enhanced vascular supply of nutrient and oxygen might reflect a malignant potential. VEGF expression and MVD 
are an important prognostic indicator in various cancers, and previous reports also indicate a significant correlation between tumor angiogenesis and invasiveness in thymic epithelial tumors $(18,19)$. Angiogenic markers such as VEGF and MVD have been already described to play an important role on the metastatic process of variable cancers $(12,18)$. However, there are a few reports on the relationship between CD98 expression and metastatic development. An experimental study demonstrated that CD98 expression is closely related with tumor cell growth of liver metastases in a rat model, and one clinicopathological study showed the role of CD98 expression in the metastatic process of variable human neoplasms $(19,24)$. In the present study, we found that CD98 expression was closely associated with angiogenesis in thymic epithelial tumors. Thymic carcinomas and high-risk thymomas have more aggressive angiogenic function than low-risk thymomas. Our results idicated that overexpression of CD98 may be necessary for tumor angiogenesis and invasiveness in thymic epithelial tumors.

Recently, some researchers described that Bcl-1 and p53 protein expression were useful markers for differentiating thymic epithelial tumors classified according to WHO classification, and high p53 has a potential prognostic importance in recurrent thymoma (16). Khoury et al described that p53 expression can predict poor outcome in thymic epithelial tumors, and Bcl-2 expression had no significant correlation with outcome (21). The present study showed that p53 was related with CD98 expression, but Bcl-2 did not correlate significantly. Although it is unclear whether the overexpression of CD98 in thymic epithelial tumors is associated with cell cycle regulators, further study is warranted.

Tumor staging has been proven to be a most important determinant of survival in thymic epithelial tumors $(25,26)$. Little is known about the useful prognostic markers other than WHO or Masaoka classification. Although the follow-up in the present study was not long enough for thymic epi-thelial tumors, we found that the overexpression of CD98 may have a potential prognostic importance in thymic epithelial tumors. Moreover, a positive CD98 expression was related with poor outcome in thymomas.

The limitation of this study must be addressed. One of the limitations is that our sample population was small, including a heterogeneous group of tumors. Another limitation is that our study includes specimens obtained through core-needle biopsy. However, only patients with an adequate specimen for immunohistochemical analysis were included. A further limitation is that the follow-up period was not long enough for thymic epithelial tumors.

In conclusion, CD98 expression was associated with the grade of malignancy in thymic epithelial tumors. Moreover, CD98 expression was closely correlated with angiogenesis and cell cycle control, and was useful for predicting poor outcome. Our results suggest that CD98 expression might be an immunohistochemical marker for malignant potential and outcome of patients with thymic epithelial tumors.

\section{Acknowledgements}

This study was supported in part by Grant 21790793 (K. K) from the Ministry of Education, Culture, Sports, Science and
Technology, Japan, and National Hospital Organization Policy Based Medical Services. We thank the staff of of pathology department in Shizuoka Cancer Center for their technical assistance in immunohistochemical analysis.

\section{References}

1. Engels EA and Pfeiffer RW: Malignant thymoma in the United States: demographic patterns in incidence and associations with subsequent malignancies. Int J Cancer 105: 546-551, 2003.

2. Rosai J and Sobin LH: Histological typing of tumours of the thymus. In: International Histological Classification of Tumours. 2nd edition. Springer, New York, pp1-59, 1999.

3. Travis WD, Brambillia E and Muller-Hermelink HK: Pathology and genetics of tumors of the lung, pleura, thymus and heart. In: WHO Classification of Tumors. IARC Press, Lyon, 2004.

4. Haynes BF, Hemler ME, Mann DL, et al: Characterization of a monoclonal antibody (4F2) that binds to human monocytes and to a subset of activated lymphocytes. J Immunol 126: 1409-1414, 1981.

5. Rintoul RC, Buttery RC, Mackinnon AC, et al: Cross-linking CD98 promotes integrin-like signaling and anchorage-independent growth. Mol Biol Cell 13: 2841-2852, 2002.

6. Takeuchi H, Kubota T, Kitai R and Kubota T: CD98 immunoreactivity in multinucleated giant cells of glioblastomas: an immunohistochemical double labeling study. Neuropathology 28: 127-131, 2008.

7. Kaira K, Oriuchi N, Shimizu K, et al: Correlation of angiogenesis with ${ }^{18} \mathrm{~F}$-FMT and ${ }^{18} \mathrm{~F}-\mathrm{FDG}$ uptake in non-small cell lung cancer. Cancer Sci 100: 753-758, 2009.

8. Kaira K, Oriuchi N, Imai H, et al: CD98 expression is associated with poor prognosis in resected non-small cell lung cancer with lymph node metastasis. Ann Surg Oncol 16: 3437-3481, 2009.

9. Kaira K, Oriuchi N, Imai H, et al: L-type amino acid transporter 1 (LAT1) and CD98 expression in the primary site and the metastatic site of human neoplasms. Cancer Sci 99: 3280-3286, 2008.

10. Kaira K, Oriuchi N, Imai H, et al: Prognostic significance of L-type amino acid transporter 1 (LAT1) and 4F2 heavy chain (CD98) expression in stage I pulmonary adenocarcinoma. Lung Cancer 66: 120-126, 2009.

11. Kaira K, Oriuchi N, Imai H, et al: Prognostic significance of L-type amino acid transporter 1 (LAT1) and 4F2 heavy chain (CD98) expression in early stage squamous cell carcinoma of the lung. Cancer Sci 100: 249-254, 2009.

12. Kaira K, Oriuchi N, Shimizu K, et al: Evaluation of thoracic tumors with ${ }^{18} \mathrm{~F}-\mathrm{FMT}$ and ${ }^{18} \mathrm{~F}-\mathrm{FDG}$ PET-CT: a clinicopathological study. Int J Cancer 124: 1152-1160, 2008.

13. Nakanishi K, Ogata S, Matsuo H, et al: Expression of LAT1 predicts risk of progression of transitional cell carcinoma of the upper urinary tract. Virchows Arch 451: 681690, 2007.

14. Monaghan $\mathrm{H}$ and William AR: The pattern of CD98 expression is different uterine serous papillary carcinoma and endometrioid endometrial carcinoma. Gynecol Oncol 104: 264-265, 2007

15. Masaoka A, Monden Y, Nakahara K and Tanioka T: Followup study of thymomas with special reference to their clinical stages. Cancer 48: 2485-2492, 1981.

16. Hiroshima K, Iyoda A, Toyozaki T, et al: Proliferative activity and apoptosis in thymic epithelial neoplasms. Mod Pathol 15: 1326-1332, 2002.

17. Weidner N, Semple JP, Welch WR and Folkman J: Tumor angiogenesis and metastasis-correlation in invasive breast carcinoma. N Engl J Med 324: 1-8, 1991.

18. Mineo TC, Ambrogi V, Baldi A, et al: Prognostic impact of VEGF, CD31, CD34, and CD105 expression and tumor vessel invasion after radical surgery for IB-IIA non-small cell lung cancer. J Clin Pathol 57: 591-597, 2004.

19. Kaira K, Oriuchi N, Imai H, et al: L-type amino acid transporter 1 (LAT1) is frequently expressed in thymic carcinomas but is absent in thymomas. J Surg Oncol 99: 433-438, 2009.

20. Mineo TC, Ambrogi V, Baldi A, Pompeo E and Mineo D: Recurrent intrathoracic thymomas: potential prognostic importance of cell-cycle protein expression. J Thorac Cardiovasc Surg 138: 40-45, 2009. 
21. Khoury T, Arshad A, Bogner P, et al: Apoptosis-related (surviving, Bcl-2), tumor suppressor gene (p53), proliferation (Ki-67), and non-receptor tyrosine kinase (SRC) markers expression and correlation with clinicopathologic variables in 60 thymic neoplasms. Chest 136: 220-228, 2009.

22. Fuchs BC and Bode BP: Amino acid transporters ASCT2 and LAT1 in cancer: partners in crime? Semin Cancer Biol 15: 254-266, 2006

23. Oxender DL and Christensen HN: Evidence for two types of mediation of neutral amino acid transport in Ehrlich cells. Nature 197: 765-767, 1963.
24. Ohkame H, Masuda H, Ishii Y and Kanai Y: Expression of L-type amino acid transporter 1 (LAT1) and 4F2 heavy chain (4F2hc) in liver tumor lesions of rat models. J Surg Oncol 78: 265-272, 2001.

25. Chen G, Marx A, Wen-Hu C, et al: New WHO histologic classification predicts prognosis of thymic epithelial tumors. Cancer 95: 420-429, 2002.

26. Kim DJ, Yang WL, Choi SS and Kim KD and Chung KY: Prognostic and clinical relevance of the World Health Organization Schema for the classification of thymic epithelial tumors. Chest 127: $755-761,2005$ 